\title{
The Importance of Using Fixed Reference Points in Studies of Collagen Crosslink Inhibition on Impeded and Unimpeded Eruption of the Rat Incisor

\author{
Norman Randall Thomas
}

Chancellor, International College of Craniomandibular Orthopedics USA, Faculty of Medicine and Dentistry, University of Alberta, Canada.

*Corresponding Author: Norman Randall Thomas, Chancellor, International College of Craniomandibular Orthopedics USA, Faculty of Medicine and Dentistry, University of Alberta, Canada.

\section{LETTER TO EDITOR}

Addition of a known lathyrogen 0.1per cent beta aminopropionitrile in the drinking water of rats produces significant differences in the unimpeded and impeded eruption rates of rat mandibular incisors compared with pair fed controls when utilizing fixed amalgam implants in the alveolar bone as reference. No significant differences are observed when the impeded tooth, occlusal plane nor gingival margin are taken as the references. The findings indicate that intrusion of the impeded reference teeth occur to an increased degree in the lathyritic condition. Given that lathyrogen reduces intramolecular and intermolecular crosslinks of collagen, it is concluded that collagen maturation clearly plays a significant role in tooth support and eruption.

\section{INTRODUCTION}

Dr John E Eastoe of the Department of Dental Science Royal College of Surgeons of England closed the seventh and final session of the Edward Colston Research Society Symposium No 27 at the University of Bristol on April $7^{\text {th }} 1975$ with the celebrated quote on the motion of heavenly bodies by Galileo Galilei referring now to the axial movements of the teeth in the process of tooth eruption: 'Er pur si muovono-And yet they move'.

In my paper 'Collagen as the Generator of tooth eruption' at that Symposium I referred to a study I had undertaken in my PhD thesis in which I had violated the principle of using fixed references in assessing the impeded and unimpeded eruption of the rodent incisor
Figure 1. In that figure the reader will observe that a diagram is presented of an experimental method of evaluation of the physiology of tooth eruption by Bryer LW. (1957) Int Dent J 7: 432-470.In that method a line $\mathrm{X}$ is cut into the tooth that is in occlusion at the start of the experiment and in 24 hours erupts and moves to $\mathrm{Y}$ distance from the occlusal plane thus attaining the rate as I=X-Y. The eruption of the unimpeded tooth cut down to the gingival margin is $\mathrm{U}=\mathrm{X}-(\mathrm{Y}+\mathrm{Z})$. It was not until several years later that I found that neither the occlusal plane, gingival margin nor the crest of the alveolar bone as seen on radiographs are acceptable fixed points for measurement of tooth eruption rates and that the eruption rates change when relevant factors are used. Studies had to be repeated using markers such as alizarin red, tetracycline fluorescence and fixed natural markers in the alveolar bone like the inferior alveolar and infraorbital nerves used as well as metallic implants for direct clinical and radiographic measurement.

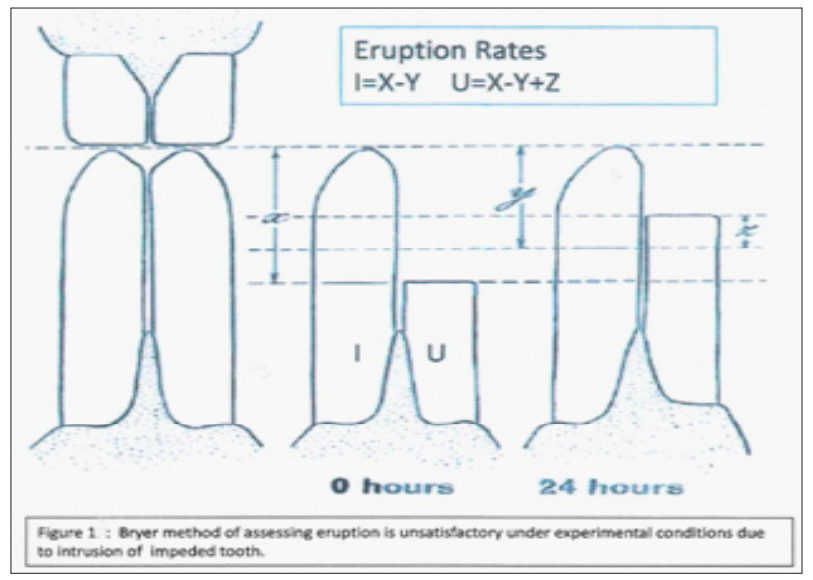

Fig1 
The Importance of Using Fixed Reference Points in Studies of Collagen Crosslink Inhibition on Impeded and Unimpeded Eruption of the Rat Incisor

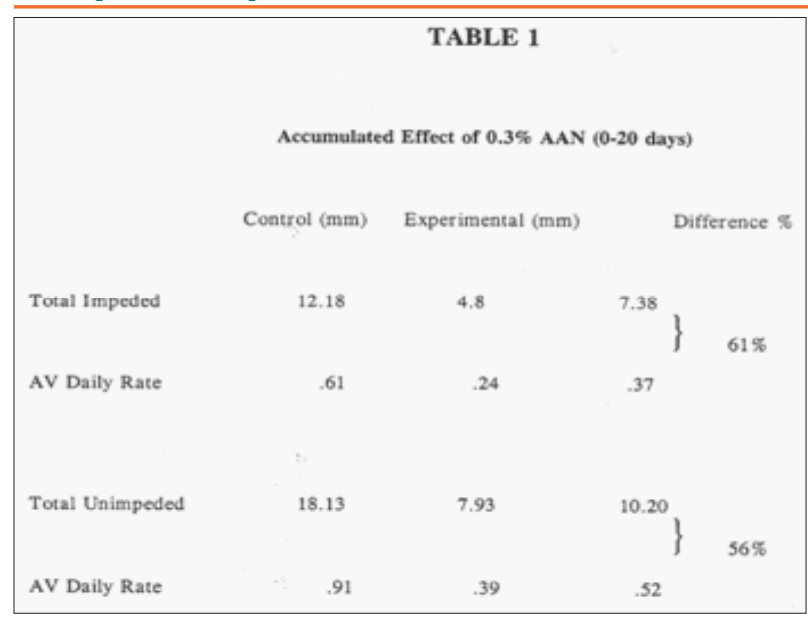

Table 1 shows the effect of the lathyrogen $0.3 \%$ Acetoaminonitrile bisulphate (AAN) on the inhibition of collagen crosslinks and hence on tooth eruption in young $54 \mathrm{gm}$. rats. The results appeared significantly positive for the hypothesis of tractional theory of collagen on tooth eruption using the Bryer (1957) technique. Drs Berkovitz BKB, Migdalski A \& Solomon M (1972) 'The effect of the lathyritic acetonitrile on the unimpeded eruption rate in normal and root resected rat lower incisors' in Archs oral Biol 17:17551763 using the same agent $0.1 \%$ AAN in the drinking water on older rats which I also had found somewhat insignificant. For these reasons and given that lathyrogen is known to reduce both intra molecular and intermolecular crosslinking of collagen as to cause bending (dilaceration) of the roots of impeded teeth from chewing hard food pellets and thus affect the measurement of tooth eruption. It was therefore decided to use the lathyrogen beta aminopropionitrile $0.1 \%$ BAPN in the drinking water of 12 older rats viz: 180-190gm. for comparison with control animals utilizing amalgam implants as fixed reference in the accompanying alveolar bone.

Table2.

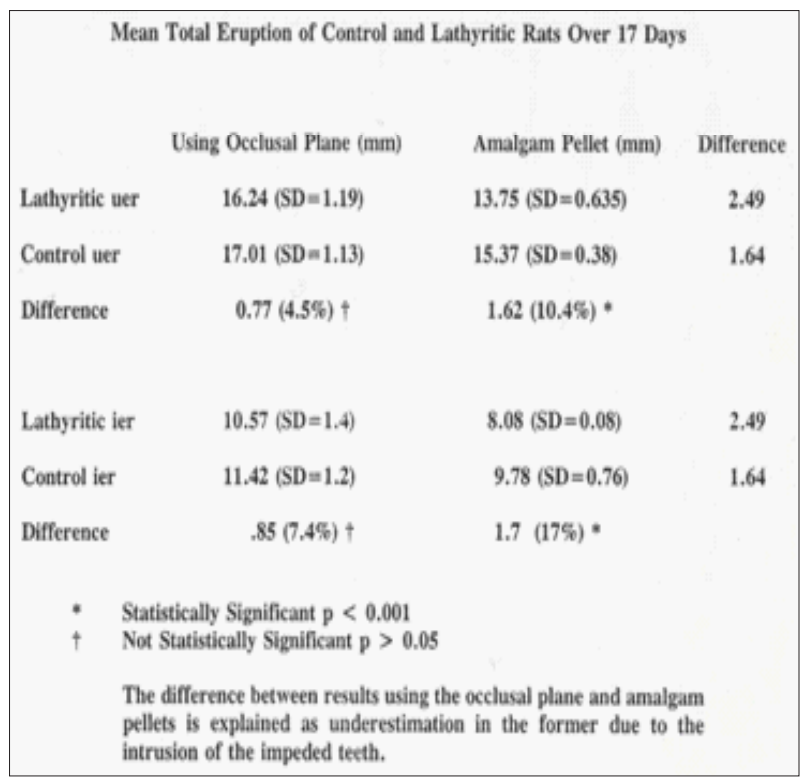

It is now clearly apparent that collagen cross-links act as agents of tractional force of eruption and as supporting mechanism in the periodontium as seen in the results of Table 2. In Table 2 Controls for impeded and unimpeded incisors erupt $0.62 \mathrm{~mm} /$ day and 1.00 $\mathrm{mm} /$ day respectively compared to $0.61 \mathrm{~mm} /$ day and $0.95 \mathrm{~mm} /$ day for lathyritic teeth when using Bryer's method of measurement compared to $0.57 \mathrm{~mm} /$ day and $0.90 \mathrm{~mm} /$ day for impeded and unimpeded controls of $0.47 \mathrm{~mm} /$ day and $0.81 \mathrm{~mm} /$ day for lathyritic teeth using metallic fixed implants respectively.

Citation: Norman Randall Thomas. The Importance of Using Fixed Reference Points in Studies of Collagen Crosslink Inhibition on Impeded and Unimpeded Eruption of the Rat Incisor. Archives of Dentistry and Oral Health. 2020; 3(2): 01-02.

Copyright: (c) 2020 Norman Randall Thomas. This is an open access article distributed under the Creative Commons Attribution License, which permits unrestricted use, distribution, and reproduction in any medium, provided the original work is properly cited. 